\title{
Multi-Objective Optimization of a Composite Rotor Blade Cross-Section
}

\author{
Swaroop B Visweswaraiah*, Hossein Ghiasi, Damiano Pasini, Larry Lessard \\ Department of Mechanical Engineering, McGill University, Canada
}

\begin{abstract}
This paper deals with the optimization of the ply angles and the internal geometry of a composite helicopter blade with a D-spar internal construction. The design involves the simultaneous optimization of several conflicting objectives such as: attaining three stiffness parameters, minimizing the blade mass and the distance between the mass-center and the aerodynamiccenter. Optimization methods with a priori and a posteriori articulation of preferences are used to solve the problem. Among the a priori approaches, the min-max approach is used to transform multiple objective functions into a single criterion which is optimized with Particle Swarm Optimization (PSO). Alternatively, the design problem is tackled using a posteriori approach by using our in-house Non-dominated Sorting Hybrid Algorithm (NSHA). The results obtained with NSHA demonstrate trade-off designs which could not be captured with the min-max approach. The multi-objective approach allows identifying a window of 10\% adjustment in mass and $20 \%$ adjustment in the distance between the mass center and the aerodynamic center with no significant deviation from the target stiffness vector. Furthermore, we have observed that the target stiffness vector can be attained more easily if the internal geometry, besides the ply angles, is considered as a design variable.
\end{abstract}

Keywords: Composite Rotor Blade, Target Vector Optimization, Multi-Objective Optimization, Pareto Front

\section{Introduction}

High stiffness-to-weight, strength-to-weight ratios and superior fatigue and dynamic properties are among crucial characteristics that a helicopter rotor blade should be designed for. Laminated composite structures can cater to such performance requirements provided that their material and geometry parameters are optimally designed. This can be a daunting task due to the interdisciplinary nature of the design problem, the presence of conflicting objectives and the number and nature of the design variables involved. This complexity has led to the use of hierarchical decomposition approaches [1] that allow modularization within the structural discipline and facilitate the interaction among hierarchical levels. The structural design of a blade is decomposed into an upper and a lower level [1,2] problems. At the upper level, the stiffness vector of the blade is treated as a variable that is adjusted to provide minimum vibration or maximum aeroelastic performance. At the lower level, which is the aim of this study, the lamination, material parameters and the cross-section geometry are optimized to provide the target stiffness vector determined at the upper level.

\footnotetext{
* swaroop.visweswaraiah@mail.mcgill.ca
} 
The cross-section design of a rotor blade requires simultaneously achieving several target stiffness parameters; however, pioneering studies are mainly focused on single-objective formulations [2]. The most popular technique is the min-max approach in which the maximum deviation from the target stiffness vector is minimized $[4,5]$. This technique yields only one solution and fails to capture other Pareto-optimal solutions. In addition, it cannot take into account other objectives, such as the structural mass and the distance between the aerodynamic center (AC) and the mass center (MC); yet, these are shown to play a crucial role in the blade performance [3]. A lower structural mass of the blade enhances the payload carrying capacity, while a shorter distance between the $\mathrm{AC}$ and $\mathrm{MC}$ facilitates reducing the pitching moments and benefits the aerodynamic performance. Leihong et al. [3] proposed to use a weighted-sum approach; however, this strategy requires assigning preferences by the user and yet fails to capture multiple optimal solutions on the Pareto front. In this study, a multi-objective optimization approach is proposed to find the Pareto front without the need to set a priori preferences on the objective functions.

After formulating the problem into a multi-objective optimization framework, an appropriate optimization algorithm must be selected. The design optimization of composite structures is often characterized by the presence of several local minima and discrete design variables. Particle Swarm Optimization (PSO) [5, 6] and Genetic Algorithms (GA) [4] are two common algorithms suitable for solving the lower-level problem of the blade design. This point was substantiated even by authors who opted to other methods, such as the method of feasible directions [7]. Computational advantage and superior performance of the PSO over GA-based approaches and gradient-based approaches have been demonstrated on particular test cases [5, 6]; however, such conclusion may not be generalized. To improve upon the high computational requirement and low convergence rate of these methods, several hybrid algorithms have been proposed [3, 7], one of the most recent ones, namely NSHA [8], is used in this research.

Finally a realistic model of the structural behavior of the blade is required. The popular approach of modeling a composite blade as a box-beam inhibits the designer to examine the sensitivity of the blade performance to the cross-sectional geometrical parameters. Although small in number, the research works that opted for a realistic model of the blade have demonstrated that ply angles and internal geometry parameters are crucial in efficient handling of the stiffness values and aerodynamic performance of a composite blade [3, 7 and 8].

This work contributes towards the optimum design of a composite blade by demonstrating a design approach that differs from those in the literature in the following four aspects: 1) it uses a blade with a realistic airfoil cross-section and a variable internal geometry, rather than a simplified box-beam model; 2) it shows the impact of the internal geometry parameters on the target stiffness vector; 3) it proposes a multi-objective optimization approach that does not require a priori prioritization of the objectives; 4) it considers mass and aerodynamic performance, besides the target stiffness vector, to clearly demonstrates the trade-off designs of the composite rotor blade.

These goals are achieved by first performing a target vector optimization using min-max formulation and PSO. In order to demonstrate the importance of the internal geometry variables and the ply angle discretization in achieving the target stiffness vector, a comparison is made 
between the results obtained with and without inclusion of the geometry variables and with different discretizations. In addition, a multi-objective formulation of the target-stiffness problem is solved using NSHA [8]. The single solution obtained using the min-max formulation is mapped on to the set of optimum solutions obtained by NSHA to demonstrate the extent of the design space that could be realized using NSHA. While the primary focus remains on the target vector optimization, simultaneous minimization of other performance parameters such as mass and the MC-AC distance are also addressed. The trade-off solutions of the Pareto front provide insight into the conflicting behavior and the interaction between the stiffness components and the aerodynamic performance parameters.

\section{Blade Modeling}

The complex geometry of the blade cross-section and the anisotropic nature of composite materials make accurate 3-D finite element modeling of a composite blade a challenging and time consuming task. As an alternative, a simplified approach can be is used to model the spatial geometry of the blade as an equivalent 1-D beam. The cross-sectional stiffness matrix is calculated based on a combination of beam theory, plate theory and classical lamination theory [10-16]. A computationally efficient in-house code is developed in MATLAB by using closedform expressions developed in [13] and a theory based on the extended Galerkin's method. The governing equations to be solved include equilibrium, constitutive equations and kinematic equations or strain-displacement relations.

The force-strain relationships for a 1-D beam is given by,

$$
\mathbf{F}=\mathbf{A}_{k} \mathbf{d}
$$

where $\mathbf{F}$ is the vector of generalized one-dimensional stress measures, $\mathbf{A}_{\boldsymbol{K}}$ represents the crosssectional stiffness matrix and $\mathbf{d}$ represents the gradient of one-dimensional displacement measures. The $4 \times 4$ cross-sectional stiffness matrix of the beam can be represented as:

$$
\left\{\begin{array}{c}
\vec{N}_{\bar{x}} \\
\vec{M}_{\bar{y}} \\
\vec{M}_{\bar{z}} \\
\vec{T}_{\bar{x}}
\end{array}\right\}=\left[\begin{array}{cccc}
E A & P_{12} & P_{13} & P_{14} \\
P_{21} & E I_{y y} & P_{23} & P_{24} \\
P_{31} & P_{32} & E I_{z z} & P_{34} \\
P_{41} & P_{42} & P_{43} & G J
\end{array}\right]\left\{\begin{array}{c}
\varepsilon_{\bar{x}}^{0} \\
1 / \rho_{\bar{y}} \\
1 / \rho_{\bar{z}} \\
\bar{v}
\end{array}\right\}
$$

The diagonal terms of the stiffness matrix are of primary importance. The first diagonal term, $E A$, represents the axial stiffness, while the others are shown in Figure 1Figure 1 . The offdiagonal terms are referred to as elastic couplings. $\vec{N}_{\bar{x}}$ in this equation represents the axial force, $\vec{M}_{\bar{y}}$ and $\vec{M}_{\bar{z}}$ are the flapwise and chordwise bending moments and $\vec{T}_{\bar{x}}$ is the torsional moment. Correspondingly, $\varepsilon_{\bar{x}}^{0}$ represents the axial strain, $\rho_{\bar{y}}$ and $\rho_{\bar{z}}$ are the curvatures around $\mathrm{Y}$ and $\mathrm{Z}$ axes and $\bar{v}$ shows the shear strain.

\section{Composite Blade Optimization}

A generic cross-section of a composite blade with a D-spar internal construction is shown in Figure 2. The design vector includes discrete and continuous variables and consisted of six ply angles $\left(\theta_{1}\right.$ to $\left.\theta_{6}\right)$, web distance from the leading edge $(w d)$, and the inclination angle of the spar web $\left(\alpha_{w e b}\right)$. To account for different manufacturing conditions, the fiber angles are discretized in 
the range of $0^{\circ}$ to $90^{\circ}$ with the increment of $10^{\circ}, 15^{\circ}$ and $45^{\circ}$. A symmetric layup is chosen in order to mitigate any warpage. The web distance, $w d$, is assumed to be continuous and ranges from $30 \%$ to $70 \%$ of the chord length so as to produce designs that are realistic and manufacturable. The spar web angle, $\alpha_{w e b}$, is also a continuous variable ranging from $-30^{\circ}$ to $30^{\circ}$. A web inclination greater than $30^{\circ}$ is not accepted as it can cause overly large resin-rich regions at the corners. The cross-sectional geometry, material properties and the target stiffness values used in this study are shown in Table 1 . The target stiffness vector is the one used by Suresh et al. [5].

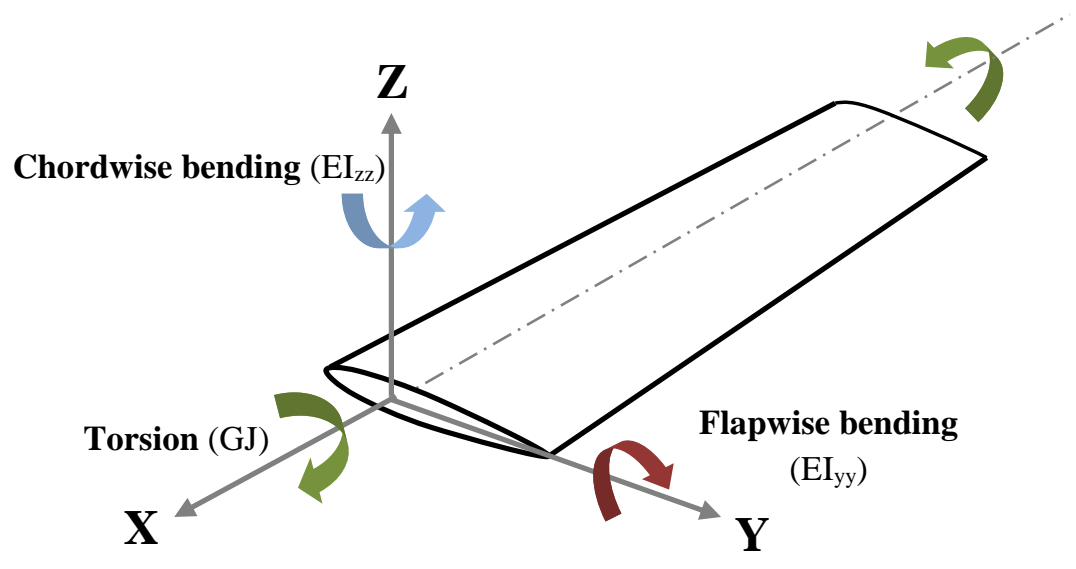

Figure 1: Composite beam and co-ordinate system

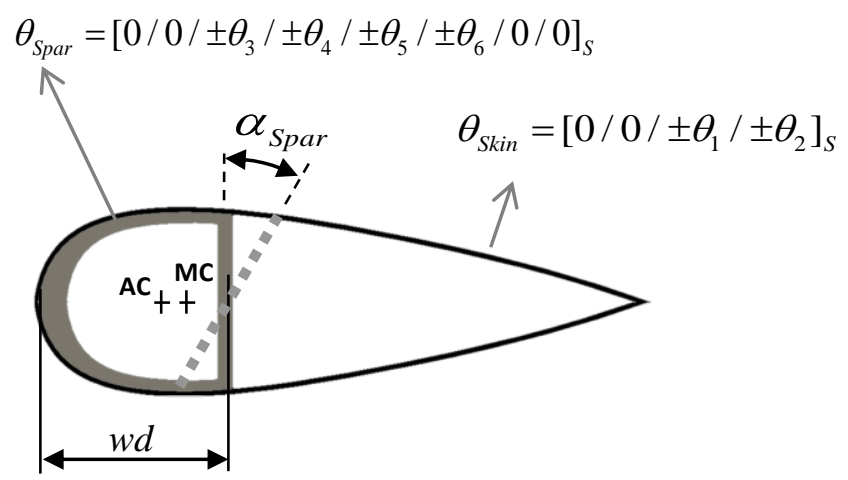

Figure 2: Generic cross-section of a rotor blade with a D-spar construction

Table 1. Airfoil geometry and Graphite/epoxy material properties, target stiffness vector

\begin{tabular}{|c|c|c|c|}
\hline $\begin{array}{l}\text { Airfoil profile } \\
\text { Chord length, } c(\mathrm{~m})\end{array}$ & $\begin{array}{c}\text { NACA0015 } \\
0.3048\end{array}$ & \multicolumn{2}{|c|}{$\begin{array}{l}\text { Material Properties of } \\
\text { Graphite/Epoxy }\end{array}$} \\
\hline Web-distance, $w d$ & $0.35 c$ & $E 1(\mathrm{GPa})$ & 141.5 \\
\hline \multicolumn{2}{|c|}{ Target Stiffness Vector [5] } & $E 2(\mathrm{GPa})$ & 9.8 \\
\hline$E I^{T}{ }_{y y}\left(\mathrm{~N}-\mathrm{m}^{2}\right)$ & 39767 & $G 12(\mathrm{GPa})$ & 5.9 \\
\hline$E I_{z z}^{T}\left(\mathrm{~N}-\mathrm{m}^{2}\right)$ about $\mathrm{AC}$ & 82916 & Poisson's ratio, $v$ & 0.42 \\
\hline$G J^{T}\left(\mathrm{~N}-\mathrm{m}^{2}\right)$ & 20420 & $\rho\left(\mathrm{kg} / \mathrm{m}^{3}\right)$ & 1445.4 \\
\hline
\end{tabular}




\subsection{Problem Formulation}

While the literature on target stiffness design of composite blades entails mainly the use of a min-max approach, here four different formulations are proposed. They are referred as Case 1 to 4.

Case 1 includes the traditional min-max approach where the objective is to minimize the maximum deviation from the target stiffness vector. The value of the min-max error represents the proximity of the stiffness vector of the current design to the target stiffness vector. Two subcases with dissimilar design variables are examined.

Case 1(a) considers only the ply angles as design variables. The web is kept constant at the vertical position at the distance of $0.35 \mathrm{c}$ (35\% of the chord length) from the leading edge of the blade. The optimization problem is mathematically represented as follows:

$$
\begin{aligned}
& \min _{\theta_{i}}\left\{\max \left\{f_{j}\left(\theta_{i}\right): \Theta^{6} \mapsto \mathfrak{R} ; i=1, \ldots, 6 ; j=1,2,3\right\}\right\} \\
& \text { S.T. }: \Theta=\left\{\theta \mid-90^{\circ} \leq \theta=10 k \text { or } 15 k \text { or } 45 k \leq 90 ; k \in \mathfrak{I}\right\}
\end{aligned}
$$

where the objective functions are defined as:

$$
f_{1}=\left(\sqrt{\frac{\left(E I_{y y}-E I_{y y}^{T}\right)^{2}}{\left(E I_{y y}^{T}\right)^{2}}} * 100\right) \quad f_{2}=\left(\sqrt{\frac{\left(E I_{z z}-E I_{z z}^{T}\right)^{2}}{\left(E I_{z z}^{T}\right)^{2}}} * 100\right) \quad f_{3}=\left(\sqrt{\frac{\left(G J-G J^{T}\right)^{2}}{\left(G J^{T}\right)^{2}}} * 100\right)
$$

Case 1(b) emphasizes the role of the internal geometry variables and considers both ply angles and the internal geometry parameters. The optimization problem here is formulated as follows:

$$
\begin{aligned}
& \min _{\theta_{i}, \alpha_{\text {spar }}, w d}\left\{\max \left\{f_{j}\left(\theta_{i}, \alpha_{\text {spar }}, w d\right):\left(\Theta^{6}, \mathfrak{R}^{2}\right) \mapsto \mathfrak{R} ; i=1, \ldots, 6 ; j=1,2,3\right\}\right\} \\
& \text { S.T. }\left\{\begin{array}{l}
\Theta=\left\{\theta \mid-90^{\circ} \leq \theta=10 k \text { or } 15 k \text { or } 45 k \leq 90 ; k \in \mathfrak{J}\right\} \\
\alpha_{\text {spar }}^{L} \leq \alpha_{\text {spar }} \leq \alpha_{\text {spar }}^{U} \\
w d^{L} \leq w d \leq w d
\end{array}\right.
\end{aligned}
$$

Case 2 describes situations where the achievement of certain components of the target stiffness vector is more important than the others; for instance, Bhadra et al. [17] showed that the bending stiffness at the root of a helicopter blade is more important than the torsional stiffness. In contrast, closer to the tip of the blade the torsional stiffness is more important than the bending stiffness. Assigning preferences to the objective functions requires information about the tradeoff among three stiffness parameters, which does not exist in practice; therefore a posteriori approach is proposed to obtain a set of optimal solutions. The formulation of the problem is similar to Case 1(b) (Equation 5) with the exception of the objective function that is defined as follows:

$\min _{\theta_{i}, \alpha_{\text {spar }}, w d}\left\{f_{j}\left(\theta_{i}, \alpha_{\text {spar }}, w d\right):\left(\Theta^{6}, \Re^{2}\right) \mapsto \Re ; i=1, \ldots, 6 ; j=1,2,3\right\}$ 
Case 3 combines the min-max target stiffness problem with another performance parameter, the blade mass. Although several researchers considered mass as an objective, none has used $a$ posteriori approach to establish the trade-off between the target stiffness and the blade mass. The formulation of the problem is similar to Equation (5) with the objective being modified as follows:

$$
\min _{\theta_{i}, \alpha_{\text {spar }}, w d}\left\{\max \left\{f_{j}\left(\theta_{i}, \alpha_{\text {spar }}, w d\right), j=1,2,3\right\}, \quad \operatorname{mass}\left(\theta_{i}, \alpha_{\text {spar }}, w d\right)\right\}:\left(\Theta^{6}, \mathfrak{R}^{2}\right) \mapsto \mathfrak{R} ; i=1, \ldots, 6
$$

Not only the blade mass, but also the distance between $\mathrm{MC}$ and $\mathrm{AC}$ is crucial in the performance of a blade. The MC-AC distance must be minimized otherwise additional non-structural mass would be required to adjust the distance. Minimization of MC-AC distance is an objective considered in the next formulation of the blade design problem, Case 4.

Case 4 is similar to Case 3 but minimizing the MC-AC distance as an objective. AC is assumed to be at $25 \%$ of the chord length.

\subsection{Optimization methods}

Among several formulations proposed in the previous section, only Case 1 converts the design problem into a single-objective optimization problem. All other formulations imply simultaneous optimization of multiple objectives. Since the relative priority of the objectives is unknown prior to solving the optimization problem, a multi-objective optimization method with a posteriori articulation of preferences is required. Such an optimization method is able to provide several optimum solutions and illustrate the trade-off among the objective functions. This section explains the single- and multi-objective optimization techniques used to solve Case 1 to 4 formulated in previous section.

\subsubsection{Particle Swarm Optimization (PSO)}

Particle Swarm Optimization [18] is a multi-agent search technique based on the behavior of a swarm. A swarm, composed of several entities called "particles", explores the design space of a problem seeking the optimum value of a single objective function. PSO is suitable for a combinatorial problem characterized by the presence of local minima and discrete design variables such as the problem in hand; however, it is limited to single-objective problem and hence Case 1 is the only formulation in this study that allows the use of this optimization method. A swarm consisted of 50 particles is found to be the minimum swarm size that return an acceptable convergence for the problem in hand. The inertia weight that controls the trade-off between the global and the local exploration ability of the swarm is linearly decreased from 0.8 to 0.1. Finally, the cognitive/acceleration constants that represent the relative importance of position of the particle to the position of the swarm are taken as 2 .

\subsubsection{Non-dominated Sorting Hybrid Algorithm (NSHA)}

To handle multiple objectives without assigning any priority or preference to the objectives, a multi-objective hybrid algorithm developed by Ghiasi et al. [8] is used. This technique, known as Non-dominated Sorting Hybrid Algorithm (NSHA), is a modified version of NSGA-II [19], which is one of the most popular multi-objective evolutionary methods due to its simplicity, effectiveness, modularity and independency on user-defined parameters [21]. Similar to other evolutionary methods, NSGA-II provides a low convergence speed compared to the traditional 
gradient-based optimization methods. NSHA combines this method with a multi-objective variation of the Nelder-Mead simplex method [20] and is shown to increase the convergence rate and to improve the spread of the solutions while maintaining the simplicity, modularity and independency to user-defined parameters [8]. In addition to handling multiple objectives without prioritization, NSHA is able to capture optimum solutions at the presence of multiple local optima and discontinuity within the design space, hence properly fitting the problem at hand.

The minimum initial population for NSHA that results in a reasonable convergence rate and preserves the diversity of the population, is found by trial-and-error and is set to 400 points. The algorithm is found to converge after approximately 50,000 function evaluations. Other userdefined parameters in NSHA are adjusted to recommended values in [8]. In both algorithms, the optimization process was initiated at random. Each case is optimized five times and the best results of all the runs are reported in the following section.

\section{Results and discussion}

The optimization algorithms described in the previous section are used to solve the four proposed cases formulated in Section 3.1. The optimum solutions are presented, contrasted and discussed in this section.

\subsection{Target stiffness design using min-max approach (Case 1)}

The min-max target stiffness design problem, formulated in Section 3.1, is solved using PSO with and without consideration of the internal geometry parameters. The results presented in Table 2 shows that the average deviation from the target stiffness vector is around $7 \%$ when the internal geometry parameters are not considered. This value is reduced to an average of $1.7 \%$ when the internal geometry parameters are considered as design variables, which clearly highlights the role of these parameters in improving elastic tailorability of the blade. A more detailed analysis of the results reveals that the stiffness vector is less sensitive to the web angle than to the web distances, which confirms the parametric studies published in [9]. The difference between the optimum cross-sectional geometry achieved in Case 1(b) and the base-line geometry in Case 1(a) is shown in Figure 3. Lower deviation from the target stiffness vector in Case 1(b) is accompanied by an increase in the spar size and consequently an undesired increase in mass. The clear trade-off between the two aforementioned objectives confirms that structural benefits can be gained if the target stiffness design is combined with the minimum mass design in a multiobjective optimization framework. Case 3 is an effort to address this issue.

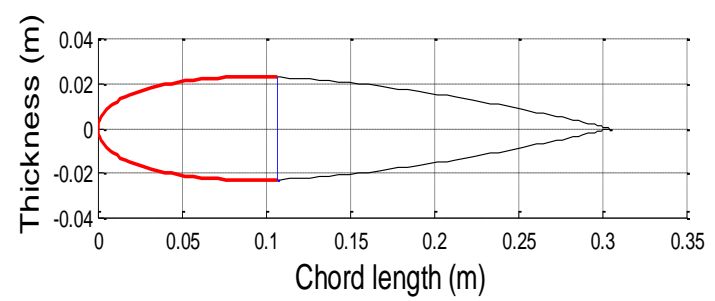

(A)

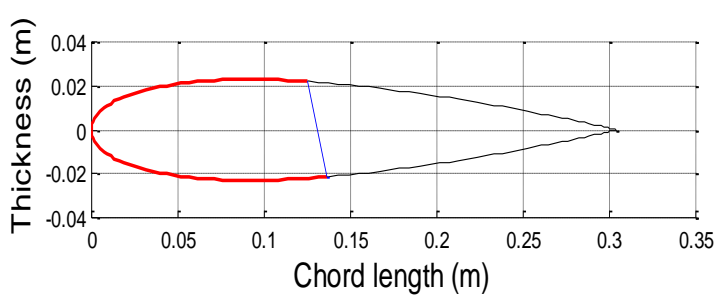

(B)

Figure 3. A) In Case 1(a) the baseline geometry yields a min-max error of 3.75; while the optimized geometry in Case 1(b) yields a min-max error of 0.11, high-lighting the effect of internal geometry parameters in reducing the min-max error. 
Table 2. Optimum solutions for Case 1(a) and 1(b)

\begin{tabular}{|c|c|c|c|c|c|c|}
\hline \multirow[b]{3}{*}{ Design variables } & \multicolumn{3}{|c|}{$\begin{array}{l}\text { Without internal geometry variables } \\
\text { Case } 1 \text { (a) }\end{array}$} & \multicolumn{3}{|c|}{$\begin{array}{l}\text { With internal geometry variables } \\
\text { Case } 1(\mathrm{~b})\end{array}$} \\
\hline & \multicolumn{3}{|c|}{ Discretization } & \multicolumn{3}{|c|}{ Discretization } \\
\hline & $10^{\circ}$ & $15^{\circ}$ & $45^{\circ}$ & $10^{\circ}$ & $15^{\circ}$ & $45^{\circ}$ \\
\hline$\theta_{1}$ (degree) & 40 & 45 & 45 & 50 & 60 & 45 \\
\hline$\theta_{2}$ (degree) & 80 & 75 & 45 & 80 & 90 & 45 \\
\hline$\theta_{3}$ (degree) & 30 & 15 & 45 & 30 & 45 & 90 \\
\hline$\theta_{4}$ (degree) & 30 & 30 & 0 & 90 & 75 & 45 \\
\hline$\theta_{5}$ (degree) & 20 & 30 & 0 & 60 & 90 & 0 \\
\hline$\theta_{6}$ (degree) & 30 & 30 & 45 & 0 & 0 & 90 \\
\hline$\alpha_{\text {spar }}($ degree $)$ & & 0 & & -7.06 & -5.63 & -0.50 \\
\hline wd as \%c & & 0.35 & & 0.43 & 0.47 & 0.43 \\
\hline Computation time $(s)$ & 151.1 & 186.9 & 276.0 & 166.4 & 247.2 & 194.1 \\
\hline Mass $(\mathrm{kg} / \mathrm{m})$ & 2.48 & 2.48 & 2.48 & 2.68 & 2.79 & 2.68 \\
\hline Objective function $(f)$ & 3.75 & 5.92 & 7.09 & 0.11 & 0.35 & 1.18 \\
\hline Number of Iterations & 35 & 42 & 50 & 41 & 60 & 46 \\
\hline
\end{tabular}

The results also show that among different discretizations schemes, the one with $10^{\circ}$ increment yielded the lowest deviation from the target stiffness vector. The level of fiber angle discretization can be evaluated as a measure representing the manufacturability of the design. From the manufacturability point-of-view, coarse ply angle discretizations are preferred to the fine ones as the placement of fibers in large number of different angles is a tedious task. Table 2 shows that fine ply angle discretizations generally return a lower deviation from the target stiffness parameters and a lower mass for the structure. Fine discretization of the ply angles is also beneficial from an optimization standpoint, as it allows better exploration of the design space and improves the convergence rate of the algorithm. The remainder of the results presented in this paper use $10^{\circ}$ increments for the ply-angles.

\subsection{Target stiffness design using NSHA (Case 2)}

While the trade-off between stiffness tailorability, blade mass and manufacturability was observed in the results of Case1, no comment could be made concerning the trade-off among the three components of the stiffness vector. Case 2 examines this trade-off by individually and simultaneously minimizing the deviation from three target stiffness values using NSHA.

Figure 4 shows the set of optimal solutions found by NSHA. This figure confirms the possibility of designing a blade that can achieve all three components of the target stiffness vector with less than $0.5 \%$ deviation from the target values. The traditional min-max approach returns only one point on the Pareto surface; however the Pareto surface shown in Figure 4 give an overview of other possible designs. This approach can help adjusting the most important stiffness parameter close to the target value with a predictable loss in other stiffness parameters; it can also be beneficial when designing an intrinsically smart composite blade with variable stiffness along the blade span. 


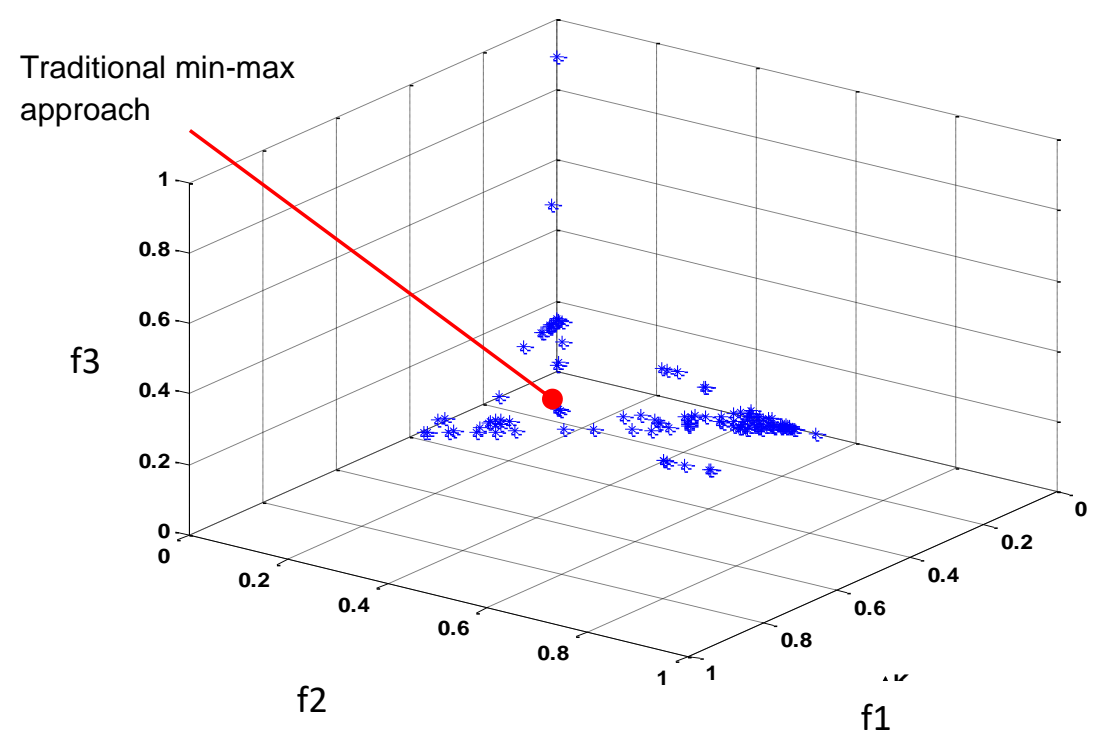

Figure 4: Pareto-front for Case 3 showing optimum solutions that represer... ...mi-offs among three target stiffness parameters. To design a structure with less than $0.4 \%$ deviation from the target value of the flapwise stiffness (f1), a penalty on other stiffness parameters is necessary.

Figure 5 shows all optimum solutions sorted by deviation from the chordwise target stiffness (f2). The majority of the solutions are concentrated at certain stiffness values, forming two distinct set of solutions marked by numbers (1) and (2) in this figure. The majority of the solutions are located in region (1) which corresponds to a very low deviation from chordwise target stiffness (f2) and relatively high deviation in other stiffness components (f1 and f3). An attempt to reduce deviation from torsional stiffness generates an unstable condition marked by drastic changes in all stiffness components. This can be attributed to a shift from one local optimum to another. The shift in local minima of $\mathrm{f} 3$ corresponds to severe changes in stacking sequence but a very small change in position and angle of the web. Traditional min-max approach can find only a single solution to this problem overlooking the solutions in region (1) and (2); however, for instance most of the solutions in region (2) exhibited lower values than this optimum with respect to f1 and f3. Using NSHA gives the opportunity to the designer to see other possible solutions and to select the optimum design according to the situation. For instance, solutions in region (1) can be better than solutions in region (2) for particular applications where torsional stiffness is more important than flapwise and chordwise bending stiffness.

From the manufacturing point of view, solutions in region (1) are more favorable because they are less sensitive to the design parameters. Region (2) is also relatively stable but less than region (1); however, it corresponds to a min-max error less than the one achievable in region (1). The final design is not only a trade-off among three target stiffness parameters but also a trade-off between manufacturability and target stiffness vector. A sensitivity analysis carried out at region (1) shows that the objectives are generally more sensitive to geometrical design variables than fiber angles, with the web distance being the most influential variable. 


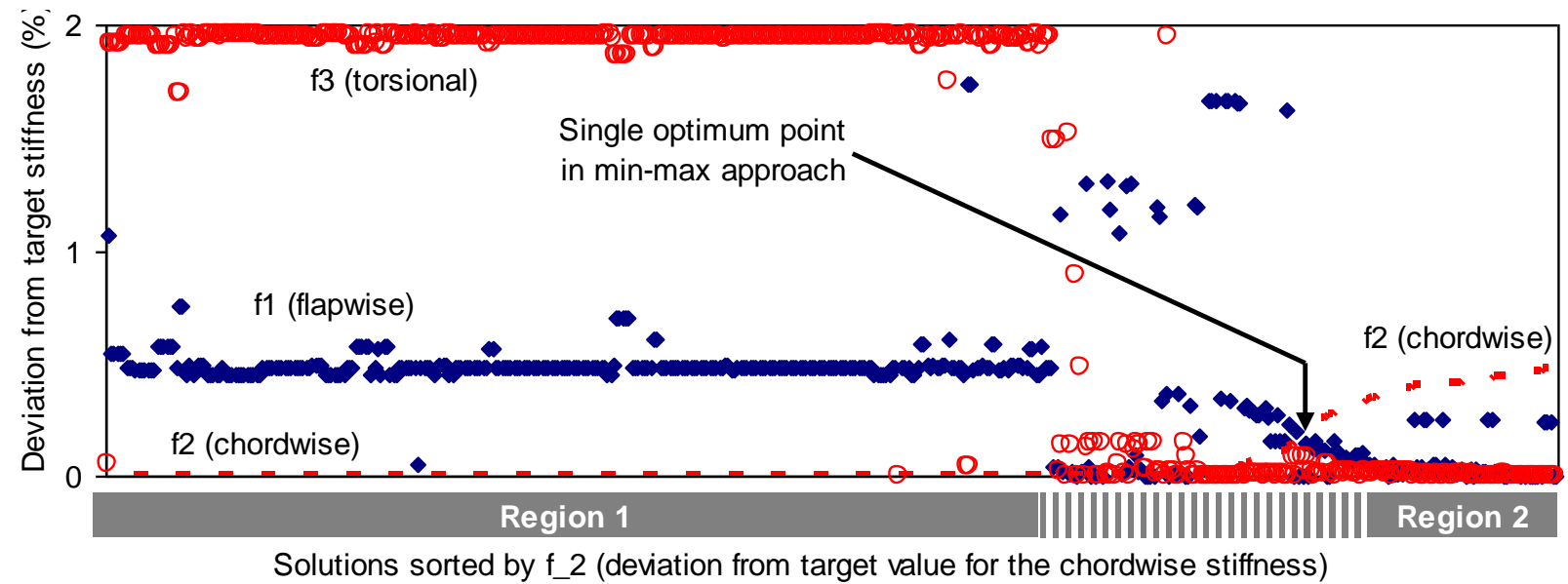

Figure 5. Comparison of the optimum solutions found by a multi-objective optimization approach and the single solution achieved by the traditional min-max approach

\subsection{Target stiffness vs. Mass (Case 3)}

The analysis of the results from Case 1 suggests that there is a trade-off between mass and level of achieving the target stiffness vector. Case 3, which is solved by NSHA, is formulated to demonstrate this trade-off. The most interesting observation is the clear exchange between mass and maximum deviation from the target stiffness values shown in Figure 6. This figure shows that for the particular blade studied here, reducing the deviation from the target stiffness from 5\% to $2 \%$ increases the mass of the blade by around 300 grams. The most influential design parameter in this trade-off is the web-distance which is also responsible for the significant increase of mass seen in Figure 6. The increase in mass corresponds to the relocation of the web from $0.45 \mathrm{c}$ to $0.37 \mathrm{c}$. The target stiffness vector is achieved with a lower error, when the web is located at the distance of approximately $0.45 \mathrm{c}$ from the leading edge; however, designs with lower masses are achieved at lower web distances. Web distance not only affects the mass, but also is crucial in the aerodynamic performance of the blade; therefore, it is necessary to see the trade-off between stiffness vector and aerodynamic performance of the blade. This relation is studied in Case 4.

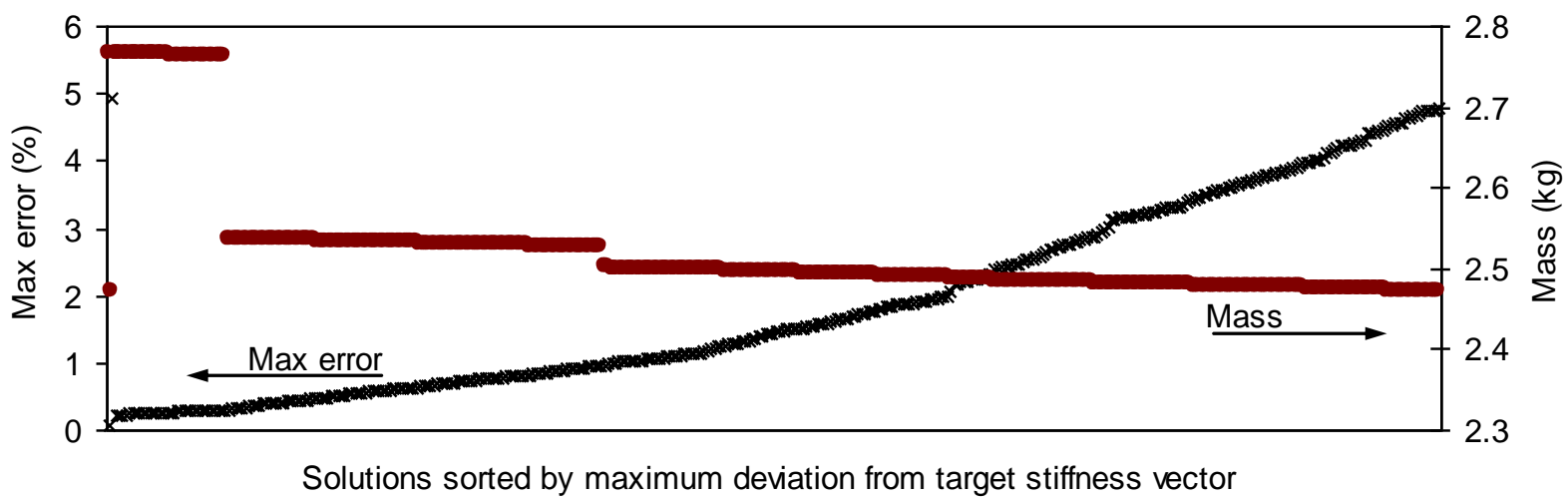

Figure 6: The trade-off shows that the traditional min-max approach, which minimizes the max-error, does not take into account the associated penalty in total mass of the blade 


\subsection{Target Stiffness vs. MC-AC distance (Case 4)}

The results of the simultaneous optimization of stiffness and aerodynamic performance are shown in Figure 7. The trade-off between MC-AC distance and the maximum deviation from target stiffness vector is apparent. Similar to Case 3, the web distance is the most influential design parameter and is responsible for the discontinuity of the data. Figure 7 also shows that a decrease in MC-AC distance from \%0.026 to \%0.022 results in only a marginal penalty in stiffness, while reducing the MC-AC distance below this value corresponds to a large deviation from the target stiffness vector (around 3\%). Since MC-AC distance is usually adjusted by adding non-structural masses, the results in Case 3 and Case 4 can be combined to design a blade with minimum total weight and maximum aerodynamic performance while meeting the assigned stiffness requirements.

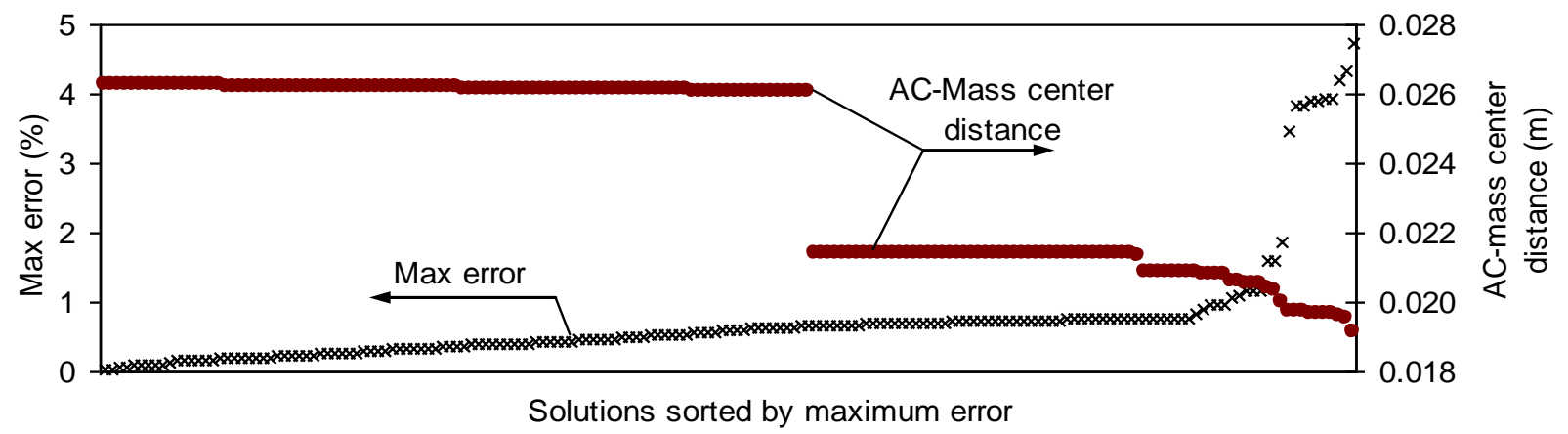

Figure 7: Total mass of the blade is in contradiction with the objective of reducing the distance between the aerodynamic center and the mass center.

\section{Conclusion}

The lower-level design optimization problem of a helicopter rotor blade has been solved using two different formulations: 1) the single-objective min-max approach for the target vector optimization utilizing Particle Swarm Optimization, 2) a multi-objective formulation utilizing multi-objective GA-based hybrid algorithm called NSHA. The multi-objective formulation involves simultaneously minimizing the structural mass of the blade, the MC-AC distance and the deviation from three target stiffness parameters. A realistic model of a blade with airfoil cross-section has been created to study the effect of internal geometry variables and ply angles on the structural and aerodynamic performance of the blade.

The first set of results obtained from a single-objective min-max optimization has demonstrated the crucial role of internal geometry parameters and ply-angle discretization on the blade stiffness. Adjustment of the internal geometry variables obtained with a $10^{\circ}$ ply-angle discretization has yielded a blade whose stiffness vector differed from the target stiffness vector by only $0.11 \%$. Further analysis of the results has shown that the internal geometry variables are more crucial than ply-angles; thus the inclusion of these parameters into the design problem greatly facilitates achieving the target stiffness vector. This study also confirmed that lowering the deviation from the target stiffness vector accompanies an increase in the blade mass, hence the simultaneous minimization of these parameters is recommended. 
In addition, multi-objective optimization of a composite blade was carried out in order to highlight the trade-offs among three stiffness components, mass and aerodynamic performance of the blade. Pareto frontiers were found for the trade-off designs among three stiffness components, mass and aerodynamic performance. The analysis of the results showed that the simultaneous consideration of mass and target stiffness can give a window of up to $11 \%$ adjustment in structural mass without a significant deviation from the target stiffness vector. This is particularly beneficial to the design of a helicopter blade, where minimizing the structural mass mitigates the dynamic stresses. Similarly, including the aerodynamic performance in the target stiffness optimization process provides opportunity to vary the MC-AC distance by up to $20 \%$; this choice can reduce the weight of non-structural mass usually added for the adjustment of the MC-AC distance.

\section{REFERENCES}

[1] Walsh JL, Young KC, Pritchard JI, Adelman HM, Mantay WR. Integrated aerodynamic/dynamic/structural optimization of helicopter rotor blades using multilevel decomposition. Technical Report 3465, NASA, Jan, 1995.

[2] Ganguli R. Survey of recent developments in Rotorcraft Design Optimization. Journal of Aircraft, Vol.41, No.3, May-June 2004.

[3] Leihong Li, Vitali V Volvoi, DH Hodges. Cross-sectional design of composite rotor blades. Journal of the American Helicopter society, July 2008.

[4] MS Murugan, S Suresh, R Ganguli, V Mani. Target vector optimization of composite box beam using realcoded genetic algorithm: a decomposition approach. Structural and Multidisciplinary Optimization (2007) 33: 131146.

[5] S Suresh, PB Sujit, AK Rao. Particle swarm optimization approach for multi-objective composite box-beam design. Composite structures, Elsevier, 81 (2007) 598-605.

[6] R Kathiravan, R Ganguli. Strength design of composite beam using gradient and particle swarm optimization. Composite Structures 81 (2007) 471-479.

[7] Paik J, Volovoi VV, Hodges DH. Cross-sectional sizing and optimization of composite blades. Proceedings of the $43^{\text {rd }}$ AIAA/ASME/ASCE/AHS/ASC Structures, Structural Dynamics, and Materials Conference (Denver, Colorado), pp. 601-610, Apr. 22-25, 2002. AIAA 2002-1322.

[8] Ghiasi H, Pasini D, Lessard L. A non-dominated sorting hybrid algorithm for multi-objective optimization of engineering problems. Engineering Optimization 43(1), pp 39-59, 2011.

[9] Visweswaraiah S, Pasini D, Lessard L. A Study of the Effect of Geometry Changes on the Structural Stiffness of a Composite D-Spar. ASME 2010 International Design Engineering Technical Conferences and Computers and Information in Engineering Conference (IDETC/CIE2010), Paper no. DETC2010-28730 pp. 51-58.

[10] Edward C Smith, Inderjit Chopra. Formulation and evaluation of an analytical model for composite box-beams. Journal of the American Helicopter society, July 1991.

[11] Berdichevsky VL. Variational-asymptotic method of constructing a theory of shells. Journal of Applied Mathematics and Mechanics (English translation of Prikladnaya Matematika i Mekhanika), Vol. 43, no. 4, pp. 664$687,1979$.

[12] Hodges DH. Non-linear composite Beam theory. AIAA, Reston, 2006.

[13] Liviu Librescu, Ohseop Song. Thin-walled composite beams - Theory and Application. Springer 2006. 
[14] Sung Nam Jung, Nagaraj VT, Chopra Inderjit. Refined structural model for thin- and thick walled composite rotor blades. AIAA Journal, Vol. 40, pp. 105-116, 2002.

[15] Laszlo P Kollar, George S Springer. Mechanics of composite structures. Cambridge University Press, 2003.

[16] Sung Nam Jung, Il-Ju Park, Eui Sup Shin. Theory of thin-walled composite beams with single and double-cell sections. Composites Part B 38, 2007, pp 182-192.

[17] Bhadra S, Ganguli R. Aeroelastic Optimization of a Helicopter Rotor Using Orthogonal Array-Based Metamodels. AIAA Journal, Vol. 44, No. 9, September 2006, pp 1941-1951.

[18] Kennedy J, Eberhart RC. Particle swarm optimization. In: Proceedings of the fourth IEEE international conference on neural networks, 1995. p. 1942-1948.

[19] Deb K, Pratap A, Agarwal S, Meyarivan T. Fast and elitist multi-objective genetic algorithm: NSGA-II. IEEE Transactions on Evolutionary Computation, 6 (2), 182-197, 2002.

[20] Nelder JA, Mead R. Simplex method for function minimization. Computer Journal, 7 (4), 308-313, 1965.

[21] Deb K. A robust evolutionary framework for multi-objective optimization. Proceeding of the $10^{\text {th }}$ Annual Conference on Genetic and Evolutionary Computation (GECCO'08), July 12-16, Atlanta, Georgia, USA, 633-640, 2008.

\section{List of Tables}

Table 1. Airfoil geometry and Graphite/epoxy material properties, target stiffness vector....................... 4

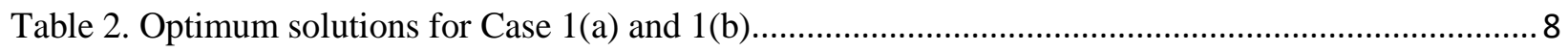

\section{List of Figures}

Figure 1: Composite beam and co-ordinate system

Figure 2: Generic cross-section of a rotor blade with a D-spar construction............................................ 4

Figure 3. A) In Case 1(a) the baseline geometry yields a min-max error of 3.75; while the optimized geometry in Case 1(b) yields a min-max error of 0.11, high-lighting the effect of internal geometry parameters in reducing the min-max error......

Figure 4: Pareto-front for Case 3 showing optimum solutions that represents trade-offs among three target stiffness parameters. To design a structure with less than $0.4 \%$ deviation from the target value of the flapwise stiffness (f1), a penalty on other stiffness parameters is necessary. ......................................... 9 Figure 5. Comparison of the optimum solutions found by a multi-objective optimization approach and the single solution achieved by the traditional min-max approach........................................................ 10 Figure 6: The trade-off shows that the traditional min-max approach, which minimizes the max-error, does not take into account the associated penalty in total mass of the blade ........................................10 Figure 7: Total mass of the blade is in contradiction with the objective of reducing the distance between the aerodynamic center and the mass center. 